\title{
molecules
}

ISSN 1420-3049

http://www.mdpi.org

\section{Applications of Microwave in Organic Synthesis: An Improved One-step Synthesis of Metallophthalocyanines and a New Modified Microwave Oven for Dry Reactions ${ }^{\dagger}$.}

\author{
Didier Villemin ${ }^{1}$, , Mohamed Hammadi², Messaoud Hachemi ${ }^{2}$ and Nathalie Bar ${ }^{1}$ \\ ${ }^{1}$ Ecole Nationale Supérieure d'Ingénieurs de Caen (ISMRA), Université de Caen, UMR CNRS 6507, \\ 6 Boulevard du Maréchal Juin F-14050 Caen Cedex, France. \\ ${ }^{2}$ Université Boumerdès, Faculté des Sciences de l'Ingénieur, Boumerdès 35000, Algeria. \\ $\dagger$ This paper was presented at the Fifth Electronic Conference on Synthetic Organic Chemistry \\ (ECSOC-5), September 1-30, 2001 (Paper E0046).
}

* Author to whom correspondence should be addressed; e-mail: didier.villemin@ismra.unicaen.fr Received: 7 September 2001 / Accepted: 10 October 2001 / Published: 31 October 2001

\begin{abstract}
Metallophthalocyanine complexes are obtained quickly and efficiently by the reaction of phthalodinitrile with hydrated metallic salts without solvent and under microwave irradiation. The use of a modified commercial microwave oven to perform this type of reactions under dry conditions is described. Metallophthalocyanines and metallododecachlorophthalocyanines of some divalent metals can be also obtained from phthalic or tetrachlorophthalic anhydrides with hydrated metallic salt and urea under microwave irradiation and without solvent.
\end{abstract}

Keywords: Phthalocyanine; microwave; complexes.

\section{Introduction}

Metallophthalocyanine complexes (PcM) are colouring materials which present high thermal stability, light fastness, and inertness to acids and alkalis. They have been studied in details for many 
years, especially with regard to their properties as pigments for printing inks and for plastics [1] and in paints and coatings. Nowadays there is renewed interest in phthalocyanine chemistry, as phthalocyanines and many of their derivatives exhibit noteworthy properties for applications in material science [2]. For example, phthalocyanines are used in laser-beam printers and photocopiers [3], in non linear optics [4], as liquid crystals [5], as photosensitizers [6], in optical data storage [7], as gas sensors [8], as electrochromic substances [9] and as carrier generation materials in near infrared (NIR) [10]. Many substituted derivatives of phthalocyanines behave like active components in various redox processes, for example, in photoredox reactions and photooxidations in solution $[2,11,12]$ and for photodynamic cancer therapy $[2,13,14]$.

We have previously reported the formation of metallophthalocyanines under microwave irradiation [15]. Recently, we have also described the preparation of metallophthalocyanines on clay, on zirconium phosphate and encapsulated in zeolites. These materials have been used as redox catalyst in organic synthesis [16]. Herein, we described the detailed work and investigation of the synthesis of metallophthalocyanines.

\section{Results and Discussion}

Microwave dielectric heating effects are used increasingly in organometallic synthesis [17]. One of the main limitations of the use of microwave ovens for synthesis can be attributed to the difficulties encountered in using organic solvents. In some cases it is possible to conduct the reaction without solvent under dry conditions as we described many years ago. This methodology is now very well established and we have performed many reactions without solvent under microwave irradiation [16]. In this paper, we describe the synthesis of metallophthalocyanines using phthalodinitrile and phthalic anhydrides as starting materials.

\section{Synthesis of metallophthalocyanines from phthalodinitrile}

Linstead and co-workers [18, 21d] first described the synthesis of metallophthalocyanines by heating a metallic salt with phthalodinitrile in a high boiling solvent. This reaction was used for the synthesis of phthalocyanine pigments like copper phthalocyanine in industry. At the beginning of our study, we used a commercial multimode microwave oven and we have performed reactions without solvent in an open device in order to avoid the typical problems associated with the use of organic solvent under microwave irradiation such as overpressure and flammability. The formation of phthalocyanines needs powerful activation (high temperature), but this fact is not a limitation because metallophthalocyanines are thermally very stable. Metallophthalocyanines were obtained by direct reaction of phthalodinitrile with hydrated metallic salt without solvent under microwave irradiation at $2450 \mathrm{MHz}$ (Scheme 1). It is not really a dry reaction because water is necessary to obtain at the beginning of the reaction a strong coupling with microwave. Water allows also the diffusion of the metallic salt and plays also a role in the redox process of formation of metallophthalocyanine. The melted phase also allows the diffusion of the salt necessary for the course of reaction and thus the phthalodinitrile can be used in excess. In some cases, ammonium formate ( 8 to 6 eq./ metal) must be 
added for promotion of the reduction of metal in the salt (Mo) or for keeping the reaction under non oxidative condition ( $\mathrm{La}, \mathrm{Ce}$ ). The excess of phthalodinitrile and the free phthalocyanine formed were easily extracted after reaction by washing the product with an organic solvent such as acetonitrile. The quantity of free phthalocyanine formed is generally very low: the infrared spectra of raw reaction mixtures showed no characteristic infrared bands corresponding to free phthalocyanine $\left(\mathrm{H}_{2} \mathrm{Pc}\right)$ (no band at $1336,1322,750$ and $700 \mathrm{~cm}^{-1}$ ).

Scheme1 : Synthesis of metallophthalocyanines under microwaves
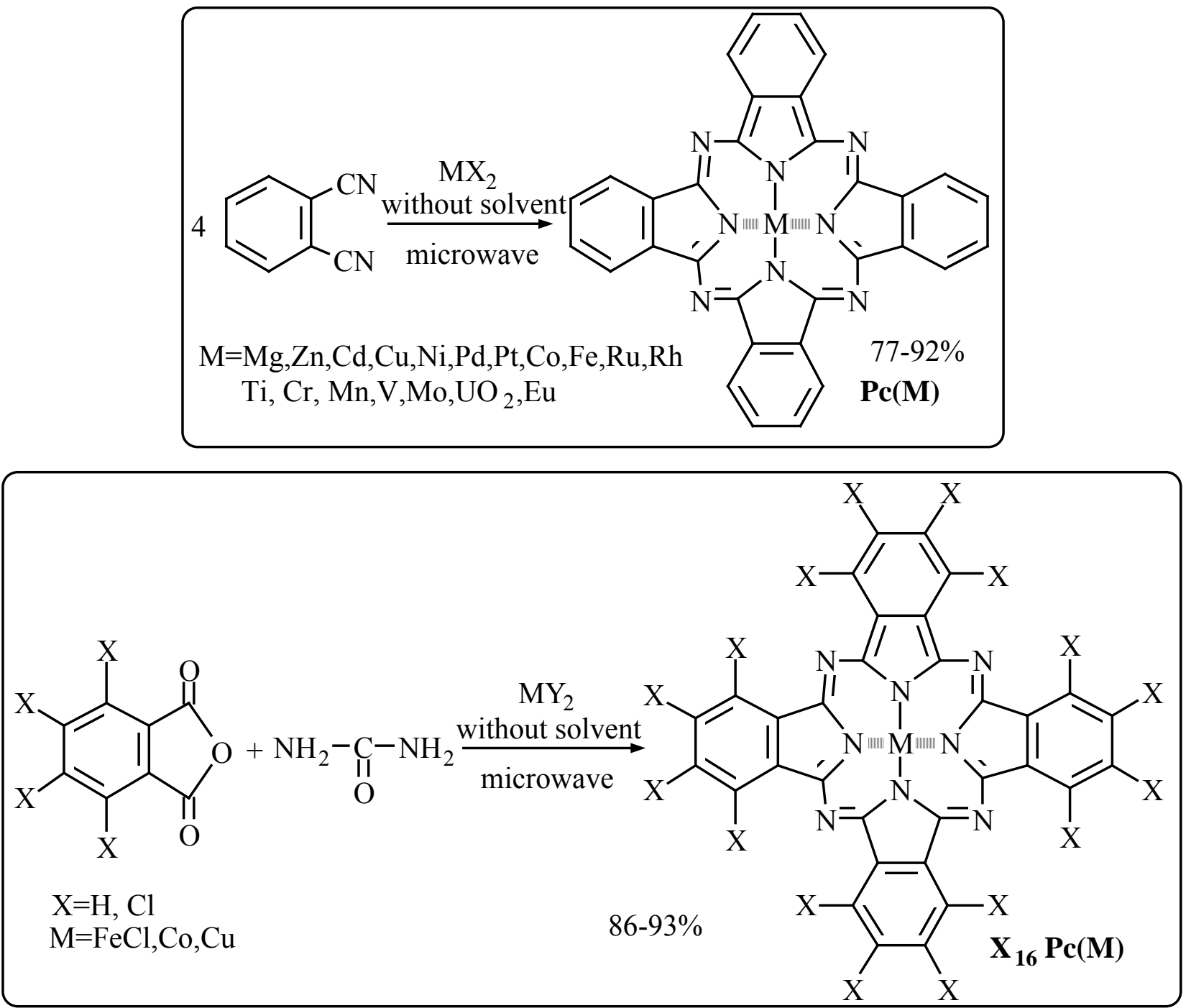

Yields of metallophthalocyanine based of the metal salt were quasi-quantitative (95-100\%). Transition metals easily gave metallophthalocyanines which are identified by their spectral data (electronic and IR spectra) and their microanalysis.

Although many of phthalocyanines were prepared with a commercial multimode oven, we found that this type of commercial oven is generally not well adapted to obtain reproducible results, so we chose to work with a modified commercial multimode oven or an industrial resonance cavity. With the purpose of camparing the results, the synthesis of copper phthalocyanine was selected as a model. 
First we have worked with a commercial multimode microwave oven (Toshiba ER 7620; max. power $630 \mathrm{~W}$ ). The use of a domestic oven has many limitations on account of the fact that the multimode cavity is calculated for irradiation of a large recipient and a sequential irradiation with full power is used. In this case, some experimental problems were encountered due to the unsatisfactory mixing of reactants and a non-uniform distribution of microwave irradiation.

In order to solve these problems, we have built a modified commercial microwave oven (Daewoo DMR 502, power $480 \mathrm{~W}$ ) in which the sample is mixed during the irradiation in order to homogenise the irradiation and to mix the reactants. This microwave oven uses a rotary evaporator equipped with an indented flask heated by a multimode cavity. The modified apparatus (Scheme 1) is in essence a rotary evaporator built within a commercial microwave oven. The reaction mixture was put in a roundbottomed indented flask shaker fitted with a frit on its top in order to prevent the powdered solids from escaping. The conduit was protected with a metallic wire mesh connected to the oven body to avoid any microwave leakages. It is also possible to conduct experiments under controlled atmosphere like nitrogen and the reaction mixtures are very efficiently mixed by the indented flask.

Scheme 2 : Modified microwave oven

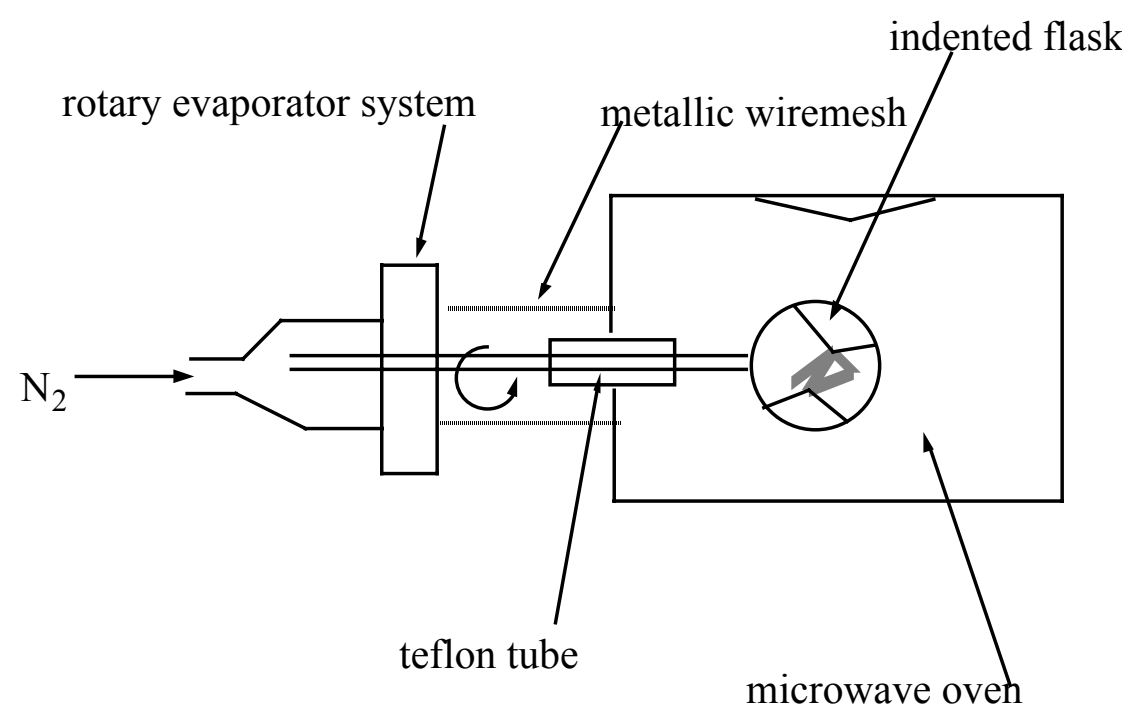

The microwaves were produced by an MES universal generator. With a small sample, we have used a $\mathrm{TE}_{013}$ monomode resonance cavity at $2450 \mathrm{MHz}$, max. working power $80 \mathrm{~W}$. The reaction was conducted in quartz tube and the solid is mixed by mechanical agitation.

\section{Choice of the mode of irradiation in the formation of $\mathrm{Pc}(\mathrm{Cu})$}

In a typical experiment, phthalodinitrile $(10 \mathrm{mmol})$ and hydrated salt $(2.5 \mathrm{mmol})$ were thoroughly mixed. The mixture was irradiated under three different modes of microwave irradiation and the results obtained are reported in Table 1. 
It should be noted that with a commercial multimode oven, the results depend upon the quantity of product, and they were very difficult to reproduce when the reactions are conducted with small samples (less than $5 \mathrm{~g}$ ). With large samples the results are poor and require a more powerful irradiation.

Table 1 : Synthesis of $\mathrm{Pc}(\mathrm{Cu})$ from phthalodinitrile $(10 \mathrm{mmol})$ and $\mathrm{Cu} \mathrm{Cl}_{2} \cdot 2 \mathrm{H}_{2} \mathrm{O}$

\begin{tabular}{|l|l|l|l|}
\hline $\begin{array}{l}\text { Microwave } \\
\text { Irradiation Sources }\end{array}$ & $\begin{array}{l}\text { Time } \\
(\mathrm{min})\end{array}$ & $\begin{array}{l}\text { Power } \\
(\mathrm{W})\end{array}$ & $\begin{array}{l}\text { Yield } \\
(\%)\end{array}$ \\
\hline microwave oven (commercial) & 10 & 560 & 92 \\
\hline modified microwave oven & 10 & 480 & 98 \\
\hline resonance cavity & 10 & 80 & 94 \\
\hline
\end{tabular}

The best results were obtained with the modified microwave oven. Its main advantages are:

- a better distribution and uniformity of the microwave irradiation

- a mixing of solid reactants during the activation with microwave irradiation.

- possibility of working under controlled atmospheres (nitrogen) and avoiding hot points.

With small samples (less than $5 \mathrm{~g}$ ) reproducible results were obtained only by the use of a resonance cavity

\section{Synthesis of metallophthalocyanines from phthalic anhydrides}

Metallophthalocyanines are often industrially prepared from the more readily available phthalic anhydrides by heating a mixture of metallic salt and phthalic anhydride in presence of urea as the source of nitrogen. Generally a molybdenum salt like ammonium molybdenate (VI) $\left[\mathrm{Mo}_{7} \mathrm{O}_{24}\left(\mathrm{NH}_{4}\right)_{6} \cdot 4 \mathrm{H}_{2} \mathrm{O}\right]$ was added in order to catalyse the reaction. The formation of phthalocyanine $[15,18]$ which requires more powerful irradiation is not easier than using phthalodinitrile. Sometimes addition of a small quantity of water was necessary due to the poor coupling of anhydride with the microwaves. Reduction of metallic salts by urea during the process lead to the formation of metal particles and some arcing were observed during the irradiation. Cracking of Pyrex or oven quartz tube during irradiation can also be observed. The reaction needs a powerful microwave intensity due to the poor adsorption of microwave radiation by the acid. In practice, the commercial microwave oven can be used, but a complex mixture of products was obtained with a bad yield of metallophthalocyanines (0-20\%) after many purification steps. The yield can be increased by the use of a modified oven, however the best yield is obtained only with the use of a resonance cavity where an efficient mixing of reactants during the reaction is observed.

This reaction is particularly interesting for the synthesis of substituted phthalocyanines because while substituted phthalodinitriles are generally not commercially available, substituted phthalic anhydrides are relatively plentiful We have illustrated this usefulness with the synthesis of hexachlorophthalocyanines, an interesting class of phthalocyanines which are very resistant to oxidation. The reaction was not general and we were unsuccessful in our attempt to prepare hexafluorophthalocyanine from tetrafluorophthalic anhydride. With mellitic anhydride, we obtained a 
similar substance to that prepared by Marvel [19], which is a mixture of polymeric phthalocyanines which can be useful as organic conductors.

\section{Conclusions}

We present a new synthesis of phthalocyanines that is rapid and efficient and allows the synthesis of a large range of metallophthalocyanine complexes. The use of a modified microwave oven allows reproducible synthesis of large quantities of phthalocyanines. The synthesis of small quantities of phthalocyanines requires the use of a focused microwave cavity. Synthesis of phthalocyanines from phthalodinitriles is easier than from phthalic anhydrides. Ammonium formate can be used when a reduction under non-oxidative conditions is required. Formation of metal particles can cause the cracking of the irradiation tube in the presence of excess of reducing agent (ammonium formate or urea in the phthalic anhydride process).

\section{Experimental}

Visible spectra $\left[\lambda_{\max } \log (\varepsilon)\right]$ were obtained in 1-chloronaphtalene (characteristic bands between

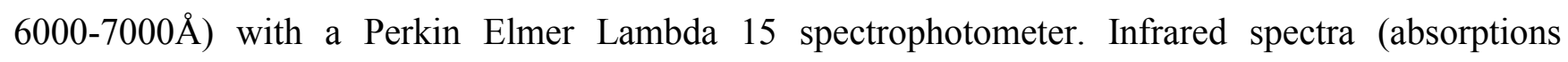
expressed in $\mathrm{cm}^{-1}$ ) were recorded on a Perkin Elmer $684 \mathrm{IR}$ spectrophotometer in $\mathrm{KBr}$. The infrared showed no free phthalocyanine $\left(\mathrm{H}_{2} \mathrm{Pc}\right)$ (i.e. no bands at 1336, 1322, 750 and $\left.700 \mathrm{~cm}^{-1}\right)$ and no presence of phthalodinitrile. Satisfactory microanalytical data $(\mathrm{C}, \mathrm{H})$ have been obtained for all metallophthalocyanine $(\operatorname{Pc}[\mathrm{M}])$ described in this paper. Activation by microwave was conducted with a commercial microwave oven (multimode) Toshiba ER 7620; power max 630W (A), or a modified multimode microwave oven (Daewoo DMR 502, power 480W) (B). A monomode resonance cavity $\mathrm{TE}_{013}$ at $2450 \mathrm{MHz}$ working with $800 \mathrm{~W}$ max. power $(\mathrm{C})$. The microwaves were produced by a MES 73-800 universal generator (Micro-ondes Energie Systèmes (MES), 2 avenue de la Cerisaie, P307, F94266 Fresnes, France). Complexes of 18 different metals were prepared (Mg, Zn, Cd, Cu, Ni, Pd, Pt, $\left.\mathrm{Co}, \mathrm{Fe}, \mathrm{Ru}, \mathrm{Rh}, \mathrm{Ti}, \mathrm{Cr}, \mathrm{Mn}, \mathrm{V}, \mathrm{Mo}, \mathrm{UO}_{2}, \mathrm{Eu}\right)$ using the commercial microwave oven. We have also prepared some complexes with the modified microwave oven and with the resonance cavity. A comparison with the first method was established.

\section{General procedures}

\section{1) Irradiation with a commercial multimode microwave oven (Method A)}

A mixture of phthalodinitrile (10 mmol, $1.28 \mathrm{~g}$ ) and finely ground hydrated metallic salt (2.5 mmol) was placed in a $25 \mathrm{~mL}$ flask. The solid was then irradiated under microwaves ( $630 \mathrm{~W}$ power) for five to fifteen minutes. The solid was washed successively with water $(15 \mathrm{~mL})$, acetone $(15 \mathrm{~mL})$ and dichloromethane $(15 \mathrm{~mL})$ and then was dried under reduced pressure. The solid was extracted by Soxhlet extraction for eight hours with acetonitrile as solvent. After drying at $110{ }^{\circ} \mathrm{C}$, the pure phthalocyanine was analyzed. 


\section{2) Irradiation with a modified multimode microwave oven (Method B)}

A mixture of phthalodinitrile (20 mmol, $2.56 \mathrm{~g}$ ) and finely ground hydrated metallic salt ( $5 \mathrm{mmol})$ was placed in a $50 \mathrm{~mL}$ flask shaker. Ammonium formate $(20 \mathrm{mmol})$ was added when a reducing medium was indicated. Water $(0.5 \mathrm{~mL})$ was added to the reaction mixtures in the case of anhydrous salts without coordinating water. The reagents were mixed and irradiated for five minutes (480 W power). The product obtained was submitted to the same workup procedure previously described.

\section{3) Irradiation in a resonance cavity (focused irradiation) (Method C)}

A mixture of hydrated metallic salt $(5 \mathrm{mmol})$ and phthalodinitrile $(10 \mathrm{mmol}, 2.56 \mathrm{~g})$ was placed in a quartz tube ( $8 \mathrm{~mm}$ diameter) under nitrogen flux. The tube was placed in the electric field of the $\mathrm{TE}_{013}$ cavity and the microwave absorbed power was set at $80 \mathrm{~W}$ for five minutes of irradiation. The product obtained was subjected to the same workup procedure as before.

Magnesium (II) phthalocyanine [Pc(Mg)] [20]

This product was obtained as a green solid from $\mathrm{MgCl}_{2}[\mathrm{P}=560 \mathrm{~W}, \mathrm{t}=5 \mathrm{~min}$, method $\mathrm{A}]$; $\mathrm{C}_{32} \mathrm{H}_{16} \mathrm{~N}_{8} \mathrm{Mg}$; Yield: 89\%; RN [1661-03-6]; Visible spectra: 6776 (3.57); 6447 (2.69); 6115 (2.78); 5835(2.30); IR: 3103, 3078 (vC-H), 3042 (vC-H), 1654, 1590, 1572, 1508 (vC-C), 1484 (vC-N), 1446, 1296 (vC-C), 1248, 1228, 1206, 966, 908 ( $\gamma \mathrm{C}-\mathrm{H}), 806,770,706(\gamma \mathrm{C}-\mathrm{H}), 562,526,474$ (ФС-С).

\section{Copper (II) phthalocyanine [Pc(Cu)] [21]}

Obtained as a blue product from $\mathrm{CuCl}_{2}[\mathrm{P}=560 \mathrm{~W}, \mathrm{t}=10 \mathrm{~min}$, method $\mathrm{A}] ; \mathrm{C}_{32} \mathrm{H}_{16} \mathrm{CuN}_{8}$; Yield: 92\%; RN [147-14-83]; Visible spectra: 6799 (3.88); 6402 (3.30); 6122 (3.39); 5810 (3.19); 5625 (3.08); IR: 3105, 3079 (vC-H), 3041 (vC-H), 1656, 1589, 1572, 1508 (vC-C), 1484 (vC-N), 1446, 1296 (vC-C), 1227, 1205, 1120, 1091, 1050 (v C-H), 965, 909 ( $\gamma \mathrm{C}-\mathrm{H}), 806,770,730,705(\gamma \mathrm{C}-\mathrm{H})$, 663, 563, 525,473 (ФС-C).

Zinc (II) phthalocyanine [Pc(Zn)] [22]

The product was obtained as a green solid from $\mathrm{ZnCl}_{2}[\mathrm{P}=490 \mathrm{~W}, \mathrm{t}=5 \mathrm{~min}$, method A]; $\mathrm{C}_{32} \mathrm{H}_{16} \mathrm{~N}_{8} \mathrm{Zn}$; Yield: 87\%; RN [14320-04-8]; Visible spectra: 6766 (3.84); 6482 (3.10); 6107 (3.13); 5858 (1.16); IR: 3104, 3082 (vC-H), 1656, 1589, 1571, 1509 (vC-C), 1472 (vC-N), 1446, 1228, 1054 $(\beta \mathrm{C}-\mathrm{H}), 903$ ( $\gamma \mathrm{C}-\mathrm{H}), 768,716(\gamma \mathrm{C}-\mathrm{H}), 562,526,473$ (ФC-C).

Cadmium (II) phthalocyanine $[P c(C d)][23]$

Obtained as a blue solid from $\mathrm{CdCl}_{2}[\mathrm{P}=630 \mathrm{~W}, \mathrm{t}=10 \mathrm{~min}$, method $\mathrm{A}] ; \mathrm{C}_{32} \mathrm{H}_{16} \mathrm{CdN}_{8}$; Yield: $88 \%$; Visible spectra: 6988 (3.78); 6767 (4.16); 6458 (3.48); 6104 (3.45); 5811(1.77); IR: 3106, 3078 (vC- 
H), 3042 (vC-H), 1654, 1590, 1572, $1484(v C-N), 1446,1296$ (vC-C), 1248, 1228, 1206, 966, 908 ( $\gamma \mathrm{C}-$ H), 806, 770, 706 ( $\gamma \mathrm{C}-\mathrm{H})$, 664, 562, 526, 474 (ФC-C).

\section{Titanium (III) phthalocyanine chloride [Pc(Ti)Cl] [24]}

Obtained as a green solid from $\mathrm{TiCl}_{3}[\mathrm{P}=630 \mathrm{~W}, \mathrm{t}=10 \mathrm{~min}$, method $\mathrm{A}] ; \mathrm{C}_{32} \mathrm{H}_{16} \mathrm{ClTiN}_{8}$; Yield: 86\%; Visible spectra: 6940 (4.30); 6820 (4.29); 6447 (3.55); 6270 (3.64); 6141 (3.50); IR: 3106, 3078 (vC-H), 3042 (vC-H), 1656, 1590, 1572, 1526 (vC-C), 1484 (vC-N), 1446, 1288 (vC-C), 1228, 1206, 1118, $1054(\beta \mathrm{C}-\mathrm{H}), 966,908(\gamma \mathrm{C}-\mathrm{H}), 806,770,706(\gamma \mathrm{C}-\mathrm{H}), 562,526,474(\Phi \mathrm{C}-\mathrm{C})$.

\section{Zirconyl phthalocyanine [Pc( $\mathrm{ZrO})][25]$}

The product was obtained as a green solid from $\mathrm{ZrOCl}_{2}[\mathrm{P}=630 \mathrm{~W}, \mathrm{t}=15 \min$, method $\mathrm{A}]$; $\mathrm{C}_{32} \mathrm{H}_{16} \mathrm{~N}_{8} \mathrm{OZr}$; Yield: 89\%; Visible spectra: 6950 (4.38); 6637 (4.08); 6321(3.87); 6167 (3.77); 6952 (3.61); 5691 (3.25); IR: 3106, 3079 (vC-H), 3041 (vC-H), 1649, 1589, 1528 (vC-C), 1484 (vC-N), 1446, 1362, 1308 (vC-C), 1228, 1208, 1090, 1054 ( $(\mathrm{C}-\mathrm{H}), 966,770,716(\gamma \mathrm{C}-\mathrm{H})$, 640, 561, 526, 473 (ФC-C).

Vanadyl phthalocyanine [Pc(VO)] [26]

The product was obtained as a blue-green solid from $\mathrm{VOCl}_{3}[\mathrm{P}=560 \mathrm{~W}, \mathrm{t}=10$ min, method $\mathrm{A}]$; $\mathrm{C}_{32} \mathrm{H}_{16} \mathrm{~N}_{8} \mathrm{OV}$; yield: 81\%; RN [13930-88-6]; Visible spectra: 7003 (4.42); 6658 (3.67); 6312 (3.69); 6023 (3.22); 5811 (1.84); IR: 3106, 3080 (vC-H), 3043 (vC-H), 3043, 1654, 1590 (vC-C), 1484 (vC-N), 1446, 1329, 1288, 1208, 1120 ( $\beta \mathrm{C}-\mathrm{H}), 1018,966,806,770,704$ ( $\gamma \mathrm{C}-\mathrm{H}), 526,473$ (ФC-С).

Molybdenyl phthalocyanine [Pc(MoO)] [27]

The product was obtained as a blue-violet solid from $\mathrm{Mo}_{7} \mathrm{O}_{24}\left(\mathrm{NH}_{4}\right)_{6} \cdot 4 \mathrm{H}_{2} \mathrm{O}[\mathrm{P}=630 \mathrm{~W}, \mathrm{t}=4 \mathrm{~min}$, method A]; $\mathrm{C}_{32} \mathrm{H}_{16} \mathrm{MoON}_{8}$; Yield: 83\%; Visible spectra: 7069 (3.77); 6717 (1.68); 6360 (1.72); 6070 (1.51); 5388 (2.60); IR: 3060 (vC-H), 1642, 1608, 1573 (vC-C), 1474 (vC-N), 1406, 1286 (vC-C), 1118, 1088, $1064(v \mathrm{C}-\mathrm{H}), 1028(\beta \mathrm{C}-\mathrm{H}), 972,912,894,858(\gamma \mathrm{C}-\mathrm{H}), 780,726(\gamma \mathrm{C}-\mathrm{H}), 622,570,506$, 468 (ФС-C).

\section{Manganese (II) phthalocyanine [Pc(Mn)] [28]}

Obtained as a grey solid from $\mathrm{Mn}\left(\mathrm{OCOCH}_{3}\right)_{2}[\mathrm{P}=560 \mathrm{~W}, \mathrm{t}=10 \mathrm{~min}$, method $\mathrm{A}] ; \mathrm{C}_{32} \mathrm{H}_{16} \mathrm{MnN}_{8}$; Yield: 90\%; RN [14325-24-7]; Visible spectra: 7262 (4.38); 6788 (3.98); 6494 (3.78); 6091 (3.48); 5740 (3.28); 5180 (3.77); IR: 3106, 3080 (vC-H), 3042 (vC-H), 1644, 1572 (vC-C), 1484 (vC-N), 1414, 1296 (vC-C), 1228, 1122, 1028 ( $\beta \mathrm{C}-\mathrm{H}), 968,906,862(\gamma \mathrm{C}-\mathrm{H}), 770,725(\gamma \mathrm{C}-\mathrm{H})$, 662, 526, 474 
Ferrous phthalocyanine $[\mathrm{Pc}(\mathrm{Fe})][29]$

Obtained as a brown solid from $\left(\mathrm{NH}_{4}\right)_{2} \mathrm{Fe}\left(\mathrm{SO}_{4}\right)_{2}[\mathrm{P}=630 \mathrm{~W}, \mathrm{t}=10 \mathrm{~min}$, method $\mathrm{A}] ; \mathrm{C}_{32} \mathrm{H}_{16} \mathrm{FeN}$; Yield: 87\%; RN [132-16-1]; Visible spectra: 6941 (3.59); 6602 (3.79); 5976 (3.52); 5276 (3.65); IR: 3106, 3078 (vC-H), 1654, 1572 (vC-C), 1478 (vC-N), 1424, 1288 (vC-C), 1124, 1025 ( $\beta C-H), 907$, $879,857$ ( $\gamma \mathrm{C}-\mathrm{H}), 770,724(\gamma \mathrm{C}-\mathrm{H}), 646,576,526,474$ (ФС-С).

Ferric phthalocyanine chloride [Pc(Fe)Cl] [29]

Obtained as a brown solid from $\mathrm{FeCl}_{3} ; \mathrm{P}=490 \mathrm{~W}, \mathrm{t}=3 \mathrm{~min}$, method $\left.\mathrm{A}\right] ; \mathrm{C}_{32} \mathrm{H}_{16} \mathrm{ClFeN}_{8}$; Yield: 79\%; RN [132-16-1]; Visible spectra: 6559 (4.08); 5976 (3.28); 5641 (2.98); 5205 (3.39).

Ruthenium phthalocyanine chloride [Pc(Ru)Cl] [30]

Obtained as a blue solid from $\mathrm{RuCl}_{3} \cdot 3 \mathrm{H}_{2} \mathrm{O} ;[\mathrm{P}=630 \mathrm{~W}, \mathrm{t}=3$ min, method $\mathrm{A}] ; \mathrm{C}_{32} \mathrm{H}_{16} \mathrm{ClN}_{8} \mathrm{Ru}$; Yield: 91\%; Visible spectra: 6515 (4.02); 600 (3.72); 5745 (3.63); 5075 (3.55); IR: 3102, 3080 (vC-H), 3036 (vC-H), 1642, 1590, 1572 (vC-C), 1482 (vC-N), 1440, 1290 (vC-C), 1228, 1206, 1102, 1064 ( $\beta C$ H), 1008, 964, 908, 891, 768, 724 ( $\gamma \mathrm{C}-\mathrm{H}), 646,571,526,474$ (ФС-С).

Nickel (II) phthalocyanine [Pc(Ni)] [31]

Obtained as a green solid from $\mathrm{NiCl}_{2}$ : $[\mathrm{P}=560 \mathrm{~W}, \mathrm{t}=10 \mathrm{~min}$, method $\mathrm{A}]$; $\mathrm{C} 32 \mathrm{H} 16 \mathrm{~N} 8 \mathrm{Ni}$; Yield: 78\%; RN [14055-02-8]; Visible spectra: 7679 (3.06); 6694 (4.00); 6447 (3.29); 6030 (3.36); 5670 (2.98); IR: 3102, 3078 (vC-H), 3042 (vC-H), 1643, 1599, 1572 (vC-C), 1484 (vC-N), 1414, 1296 (vCC), 1228, 1208, 1089, 1031( $\beta \mathrm{C}-\mathrm{H}), 966,895,857$ ( $\gamma \mathrm{C}-\mathrm{H}), 772,570,528,474$ (ФC-C).

Palladium (II) phthalocyanine [Pc(Pd)] [32]

Obtained as a blue solid from $\mathrm{PdCl}_{2} ;[\mathrm{P}=630 \mathrm{~W}, \mathrm{t}=10 \mathrm{~min}$, method $\mathrm{A}] ; \mathrm{C}_{32} \mathrm{H}_{16} \mathrm{~N}_{8} \mathrm{Pd}$; Yield: 65\%; Visible spectra: 7058 (2.98); 6610 (3.85); 6329 (3.15); 5969 (3.21); IR: 3102, 3080 (vC-H), 3040 (vC-H), 1676, 1590, 1572 (vC-C), 1484 (vC-N), 1412, 1296 (vC-C), 1228, 1208, 1124, 966, 928, 843 $(\gamma \mathrm{C}-\mathrm{H}), 770,662,572,526,474$ (ФС-C).

Platinum (II) phthalocyanine [Pc(Pt)] [33]

Obtained as a green solid from $\mathrm{K}_{2} \mathrm{PtCl}_{4}$ : $[\mathrm{P}=560 \mathrm{~W}, \mathrm{t}=10$ min, method $\mathrm{A}] ; \mathrm{C}_{32} \mathrm{H}_{16} \mathrm{~N}_{8} \mathrm{Pt}$; Yield: 78\%; Visible spectra: 6941 (2.74); 6498 (3.57); 6258 (3.09); 5875 (3.10); IR: 3106, 3080 (vC-H), 3042 (vC-H), 1656, 1590, 1572 (vC-C), 1484 (vC-N), 1412, 1298 (vC-C), 1228, 1206, 1127, 1043 ( $\beta C-H)$, 966, 928, 867 ( $\gamma \mathrm{C}-\mathrm{H}), 770,664,574,526,474$ (ФС-C). 
Cobalt (II) phthalocyanine [Pc(Co)] [34]

Obtained as a violet solid from $\mathrm{CoCl}_{2} \cdot 6 \mathrm{H}_{2} \mathrm{O}[\mathrm{P}=560 \mathrm{~W}, \mathrm{t}=10$ min, method A]; C32H16CoN8; Yield: 91\%; RN [3317-67-7]; Visible spectra: 6698 (4.47); 6400 (3.94); 6043 (3.85); IR: 3079 (vC-H), 3040 (vC-H), 1657, 1609 (vC-C), 1469 (vC-N), 1425, 1288 (vC-C), 1052 ( $\beta \mathrm{C}-\mathrm{H}), 913,868(\gamma \mathrm{C}-\mathrm{H})$, $805,772,722(\gamma \mathrm{C}-\mathrm{H}), 572,517,473$ (ФC-C).

\section{Rhodium (III)phthalocyanine chloride [Pc(Rh)Cl] [35]}

Obtained as a blue-green solid from $\mathrm{RhCl}_{3} \cdot 3 \mathrm{H}_{2} \mathrm{O}[\mathrm{P}=490 \mathrm{~W}, \mathrm{t}=5$ min, method $\mathrm{A}]$; $\mathrm{C}_{32} \mathrm{H}_{16} \mathrm{ClN}_{8} \mathrm{Rh}$; Yield: 87\%; Visible spectra: 6549 (4.15); 6282 (3.51); 5907 (3.56); IR: 3100, 3082 (vC-H), 3036 (vC-H), 1664, 1618 (vC-C), 1482 (vC-N), 1454, 1298 (vC-C), 1228, 1206, 1076 ( $\beta \mathrm{C}-\mathrm{H})$, 966, 916, $882(\gamma \mathrm{C}-\mathrm{H}), 768,704$ ( $\gamma \mathrm{C}-\mathrm{H})$ 664, 560, 526, 474 (ФС-С).

Europium (II) phthalocyanine [Pc(Eu)] [36]

The product was obtained as a green solid from $\mathrm{Eu}_{2} \mathrm{O}_{3}[\mathrm{P}=630 \mathrm{~W}, \mathrm{t}=15$ min, method $\mathrm{A}]$; $\mathrm{C}_{32} \mathrm{H}_{16} \mathrm{EuN}_{8} \mathrm{O}$; Yield: 77\%; Visible spectra: 6929 (3.96); 6754 (4.14); 6352 (3.76); 6098 (3.79); IR: 3080 (vC-H), 3037 (vC-H), 1590 (vC-C), 1484 (vC-N), 1452, 1296 (vC-C), 1228, 1206, 1054 ( $\beta C-H)$, 966, 912, $870(\gamma \mathrm{C}-\mathrm{H}), 806,770,706(\gamma \mathrm{C}-\mathrm{H}), 560,526,474$ (ФС-С).

\section{Uranyl phthalocyanine [Pc(UO] [37]}

Obtained as a brown solid from $\mathrm{UO}_{2}\left(\mathrm{C}_{2} \mathrm{H}_{4} \mathrm{O}_{2}\right)_{2} \cdot 2 \mathrm{H}_{2} \mathrm{O}[\mathrm{P}=630 \mathrm{~W}, \mathrm{t}=5$ min, method A]; $\mathrm{C}_{32} \mathrm{H}_{16} \mathrm{~N}_{8} \mathrm{UO}_{2}$; Yield: 91\%; Visible spectra: 6977 (3.45); 6776 (3.41); 6642 (3.47); 6329 (3.91); 6094 (3.83); IR: 3106, 3080 (vC-H), 3042 (vC-H), 1642, 1590 (vC-C), 1484 (vC-N), 1452, 1298 (vC-C), 1228, 1206, 1054 (ßC-H) 964, 916, 806, 770, 705 ( $\gamma \mathrm{C}-\mathrm{H})$, 684, 562, 526, 474 (ФC-C).

Lanthanum (III) phthalocyanine chloride [Pc(La)Cl] [38]

Obtained as a green solid from $\mathrm{LaCl}_{3}$ and $\mathrm{HCO}_{2} \mathrm{NH}_{4}(6 \mathrm{eq}) ; \mathrm{C}_{32} \mathrm{H}_{16} \mathrm{ClLaN}_{8}$; Yield: $87 \%$; Visible spectra: 6976 (3.88); 6637 (3.57); 6353 (3.29); 6053 (3.09); IR: 3138, 3024 (vC-H) 1575 (vC-C) 1426, $1088(\beta \mathrm{C}-\mathrm{H}), 966,780,706(\gamma \mathrm{C}-\mathrm{H}), 668,582,526,474$ (ФC-C).

\section{Chromium (II) phthalocyanine $[\mathrm{Pc}(\mathrm{Cr})][39]$}

Obtained as a green solid from $\mathrm{CrO}_{3}$ and $\mathrm{HCO}_{2} \mathrm{NH}_{4}$ (8 eq); $\mathrm{C}_{32} \mathrm{H} 16 \mathrm{CrN} 8$; Yield: $92 \%$; Visible spectra: 6842 (4.42); 6629 (4.83); 6299 (4.18); 6063 (3.99); 5730 (3.63); IR: 3106, 3078 (vC-H), 3042 (vC-H), 1684, 1610, 1560 (vC-C), 1488 (vC-N), 1448, 1298 (vC-C), 1234, 1058 (ßC-H), 942, 893, 856 $(\gamma \mathrm{C}-\mathrm{H}), 814,756,718(\gamma \mathrm{C}-\mathrm{H}), 688,548,526,472$ (ФС-С). 
Cerium (III) phthalocyanine chloride [Pc(Ce)Cl] [40]

The product was formed as a green solid from $\mathrm{CeCl}_{3} \cdot 7 \mathrm{H}_{2} \mathrm{O}$ and $\mathrm{HCO}_{2} \mathrm{NH}_{4}\left(8\right.$ eq); $\mathrm{C}_{32} \mathrm{H}_{16} \mathrm{CeClN}_{8}$; Yield: 90 \%; Visible spectra: 7017 (3.77); 6851 (3.96); 6619 (3.51); 6198 (3.22); IR: 3142, 3080

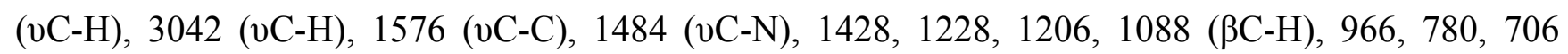
$(\gamma \mathrm{C}-\mathrm{H}), 670,526,473$ (ФС-C).

\section{From phthalic anhydride: General procedure}

A mixture composed of tetrachlorophthalic anhydride $(10 \mathrm{mmol})$ and urea $(20 \mathrm{mmol})$ was ground in a mortar in the presence of $\mathrm{Mo} 7 \mathrm{O} 24(\mathrm{NH} 4) 6 \cdot 4 \mathrm{H} 2 \mathrm{O}(0.1 \mathrm{mmol})$, then the appropriate metallic salt $(5 \mathrm{mmol})$ was added. The reaction mixture was put in a tube and activated under microwave irradiation in a resonance cavity (method $\mathrm{C})$. The final product was taken up again with $1 \mathrm{~N} \mathrm{HCl}(50 \mathrm{~mL})$ then neutralised with $1 \mathrm{~N}$ sodium hydroxide $(50 \mathrm{~mL})$ and washed with water. The solid dried at $110^{\circ} \mathrm{C}$ was then purified by Soxhlet extraction with acetonitrile for eight hours. The phthalocyanine obtained was identified with spectroscopic methods. The following phthalocyanines were obtained from phthalic anhydride: $\mathrm{Pc}(\mathrm{Cu})$; $\mathrm{Pc}(\mathrm{Co})$; $\mathrm{Pc}(\mathrm{MoO})$ 93\%, [P=560W, t= 5min., method $\mathrm{C}]$.

From tetrachlorophthalic anhydride:

Iron III Phthalocyanine chloride $\left[\mathrm{Cl}_{16} \mathrm{Pc}(\mathrm{FeCl})\right][29]$

The product was obtained as a green solid from tetrachlorophthalic anhydride (17 mmol; $4.81 \mathrm{~g})$ and $\mathrm{FeCl}_{3}(0.83 \mathrm{~g}) ; \mathrm{C}_{32} \mathrm{Cl}_{17} \mathrm{FeN}_{8} ;[\mathrm{P}=420 \mathrm{~W}, \mathrm{t}=2 \mathrm{~min}$, method C]; Yield: $86 \%$; Visible spectra: 685, (4); 656, (4); 611, (7); IR: 1664, 1610 (vC-C), 1558 (vC-C), 1436 (vC-C), 1264, 1214, 1192, 1134, 1098, 954, 930, 768 (vC-Cl), 738, 668, 648, 590 (vC-Cl), 510, 470 (Ф C-C).

Cobalt (II) phthalocyanine chloride $\left[\mathrm{Cl}_{16} \mathrm{Pc}(\mathrm{Co})\right][34]$

Obtained as a green solid from tetrachlorophthalic anhydride $(17 \mathrm{mmol} ; 4.81 \mathrm{~g})$ and $\mathrm{CoCl}_{2} \cdot 6 \mathrm{H}_{2} \mathrm{O}$ $(0.93 \mathrm{~g})$; $\mathrm{C} 32 \mathrm{Cl} 16 \mathrm{CoN} 8 ;[\mathrm{P}=620 \mathrm{~W}, \mathrm{t}=2 \mathrm{~min}$, method C); Yield $92 \%$; Visible spectra: 698.1; 667.9; 642.0; 640.0; 604.3; IR: 1676, 1610 (vC-C), 1430 (vC-C), 1272, 1212, 1192, 1132, 1098, 928, 898, 816, 768 (vC-Cl), 736, 658, 592 (vC-Cl), 532, 468 (ФC-C).

\section{Phthalocyanatocopper chloride $\left[\mathrm{Cl}_{16} \mathrm{Pc}(\mathrm{Cu})\right][21]$}

Obtained as a blue-green solid from tetrachlorophthalic anhydride (17 mmol, $4.81 \mathrm{~g})$ and $\mathrm{CuCl}_{2} \cdot 2 \mathrm{H}_{2} \mathrm{O}(0.85 \mathrm{~g}) ; \mathrm{C}_{32} \mathrm{Cl}_{16} \mathrm{CuN}_{8} ;[\mathrm{P}=620 \mathrm{~W}, \mathrm{t}=4 \mathrm{~min}$, method $\mathrm{C})$; Yield $93 \%$; Visible spectra: 707.9; 668.2; 652.3; 603.2; 583.4; IR: 1611 (vC-C), 1495, 1392 (vC-C), 1275 (vC-C), 1212, 1194, 1097, 948, 928, 805, 768 (vC-Cl), 657, 620, 591 (vC-Cl), 510. 
From tetrafluorophthalic anhydride:

Under similar conditions, no phthalocyanine was obtained with either $\mathrm{CuCl}_{2}, \mathrm{CoCl}_{2}$ or $\mathrm{MnCl}_{2}$.

\section{References and Notes}

1. (a) Moser, F.H.; Thomas, A.L. The Phthalocyanines, CRC Press: Boca Raton, FL, 1983; (b) Bezerin, B.D. Coordination Compounds of Porphyrins and Phthalocyanines, J. Wiley and Sons: New York, NY, 1981; (c) Bauman, F.; Bienert, B.; Rösch, G.; Vollman, H.; Wolf, W. Angew. Chem., 1956, 68, 133.

2. (a) Leznoff, C.C.; Lever, A.B.P., Phthalocyanines, Properties and Applications, VCH Publishers: New-York. 1989-1993, 1-3; (b) Hanack, M.; Lang, M. Adv. Mater. 1994, 6, 819.

3. (a) Borsenger, P.M.; Weiss, D.S. Organic Photoreceptors for Imaging System. Marcel Dekker, Inc.: New York, 1993; (b) Law, K.Y. Chem. Rev. 1993, 93, 449.

4. (a) Casstevens, M.; Samok, M.; Pfleger, J.; Prasard, P.N. Chem. Phys. 1990, 92, 2019; (b) Simon, J.; Bassoul, P.; Norvez, S. New J. Chem. 1994, 13, 13.

5. (a) Van Der Pol, J. F.; Neeleman, E.; Zwikker, J.W.; Nolte, R.J.M.; Drenth, W.; Aerts, J.; Visser, R.; Picken J. J. Liq. Cryst. 1989, 6, 577; (b) Simon, J.; Sirlin, C. Pure Appl. Chem. 1989, 61, 1625.

6. Kato, M.; Nishioka, Y.; Kaifu, K.; Kawamura, K.; Ohno, S. Appl. Chem. Lett. 1985, 86, 196.

7. Kuder, J. E. J. Imag. Sci. 1988, 32, 51.

8. (a) Temofonte,T. A.; Schoch, K.F. J. Appl. Phys. 1989, 65, 1350; (b) Sadaoka, Y.; Jones, T.A.; Göpel, W. Sensors Actuators B. 1990, I, 148.

9. Riou, M.T.; Clarisse, C.J. Electroanal. Chem. 1988, 249, 181.

10. Law, K.Y. Chem. Rev. 1993, 93, 449.

11. Darwent, J.R.; Douglas, P.; Harriman, A.; Porter, G.; Richoux, M.C. Coord. Chem. Rev. 1982, 44, 83.

12. Wöhrle, D.; Gitzel, J.; Krawczyk, G.; Tsuchida, E.; Ohno, H.; Nishisaka, T.J. Macromol. Sci. C. A. 1988, 25, 1227.

13. Henderson, B.A.; Dougherty, T.J. Photochem. Photobiol. 1992, 55, 145.

14. Wöhrle, D.; Ardeschirpur, A.; Heuermann, A.; Muller, S.; Graschew, G.; Rinneberg, H.; Kohl, M.; Neukammer, J. Makromol. Chem. Makromol. Symp. 1992, 59, 17.

15. Our work on synthesis of metallophthalocyanines was partially described in a monograph and in a communication: (a) Bram, G.; Loupy, A.; Villemin, D., Solid Supports and Catalysts in Organic Synthesis, K. Smith, editor; Ellis Horwood and Prentice Hall: Chichester, U.K., 1992, 12, 302-326; (b) Villemin, D; Hammadi, M. Actes of 3 ème Colloque Franco-Maghrebin de Catalyse, CFMC, 1996, 622.

16. Villemin, D.; Hammadi, M.; Hachemi, M. Synth. Commun., 2001, in press.

17. (a) Kingston, H. M.; Haswell, S. J. (eds), Microwave-Enhanced Chemistry. Fundamentals, Sample Preparation, and Applications, American Chemical Society: Washington, D.C., 1997; (b) Varma, R.S. Green Chem. 1999, 43.

18. Shaabani, A. J. Chem. Res. 1998, 672. 
19. Marvel , C.S.; Rassweiler, J. J. Amer. Chem. Soc. 1958, 80, 1197.

20. (a) Linstead, R.P.; Robertson, J.M. J. Chem. Soc. 1936, 173; (b) Lexas, D.; Reix, M. J. Chim. Phys. Physicochim. Biol. 1974, 71, 517. (c) Lhost, J.M; Grivet, J.Ph. Adv. Radiat. Res. Phys. Chem. 1973, 327-1. (d) Shablya, A.V.; Terenin, A.N. Optika Spectroskopiya. 1960, 9, 533 [C. A. 1961, 55, 12028i]; (e) Seybold, P.G.; Gouterman, M. J. Mol. Spectrosc. 1939, 31, 1.

21. (a) Linstead, R.P. J. Chem. Soc. 1934, 1010; (b) Dent, C.E.; Linstead, R.P. J. Chem. Soc. 1934, 1027; (c) Linstead, R.P.; Lowe, A.R. J. Chem. Soc. 1934, 1031; (d) Linstead, R.P. J. Chem. Soc., 1934, 1016; (e) Barrett, P.A; Dent, C.E.; Linstead, R.P. J. Chem. Soc. 1936, 1720; (f) Linstead, R.P.; Robertson, J.M. J. Chem. Soc. 1936, 1736; (g) Berezin, B.D. Izv.Vyssh. Ucheb. Zaved., Khim. Khim. Tekhnol. 1968, 11, 537-541. C. A. 1968, 69, 72393 c. (h) Takashi, O.; Michiakazu , N.; Atsushi, K.; Shuji, F., Japan Catalytic Chemical Industry Co., Ltd. Japan Pat. 1970, 70, 07,659. [C.A. 1970, 73, 16287].

22. (a) Seybold, P.G.; Gouterman, M. J. Mol. Spectrosc. 1969, 31, 1; (b) Barrett, P.A.; Dent, C.E.; Linstead, R.P. J. Chem. Soc. 1936, 1719. (c) Vacus, J.; Memetzidis, G.; Doppelt, P.; Simmon, J. J. Chem. Soc. Chem. Commun. 1994, 697.

23. Lexas, D.; Reix, M. J. Chim. Phys. Physicochim. Biol. 1974, 71, 517.

24. (a) Block, B.P.; Meloni, E.G. Inorg. Chem. 1965, 4, 111. (b) Taube, R.Z. Chem., 1963, 3, 194.

25. Plyushchev, V.E.; Shkolner, L.P.; Rodin, I.A. Zh. Neorgan. Khim. 1964, 9, 125; Mühler, P. Z. Chem. 1967, 1, 352.

26. (a) Mitsuo, S.; Takao, K. Chem. Pharm. Bull. 1968, 16, 2517. (b) Berezin, B.D. Izv.Vyssh. Ucheb. Zaved., Khim. Khim. Tekhnol. 1968, 11, 537-541 [C.A. 1968, 69, 72393c].

27. James, S.A.; Ray, A.K; Silver, J. Phys. Stat. Sol. 1992, 129, 435.

28. (a) Lever, A.B.P. J. Chem. Soc. 1965, 1821; (b) Barrett, P.A.; Dent, C.E.; Linstead, R.P. J. Chem. Soc. 1936, 1719; (c) Rutter, H.A.; Mc Queen, J.D. J. Inorg. Nucl. Chem. 1960, 12, 361; (d) Mason, R.; Willems, G.A.; Fielding, P.E. J. Chem. Soc. Dalton Trans 1991, 676.

29. (a) Linstead, R.P. Chem. Soc. 1934, 1016; (b) Linstead, R.P.; Robertson, J.M. J. Chem. Soc. 1936, 1736; (c) Barrett, P.A.; Dent, C.E.; Linstead, R.P. J. Chem. Soc. 1936, 1719; (d) Barrett, P.A.; Frye, D. A.; Linstead, R.P. J . Chem. Soc. 1938, 1157; (e) Metz, J.; Schneider, O.; Hanack, M. Inorg. Chem. 1984, 23, 1065; (f) Rudenko, A.P.; Dobrosel'skaya, N.P. Zh. Obshch. Khim. 1961, 31, 3667 [C. A. 1962, 57, 9856b]; (g) Taube, R.; Drevs, H.; Fluck, E.; Kuhn, P.; Brauch, K.F. Z. Anorg. Allg. Chem. 1969, 364, 297.

30. Krueger, P.C.; Kenney, M.E. J. Inorg. Nucl. Chem. 1963, 25, 303.

31. (a) Enokida, T.; Hirohashi, R. Chem. Letters. 1991, 2155; (b) Berezin, B.D. Izv.Vyssh. Ucheb. Zaved. Khim. Khim. Tekhnol. 1968, 11, 537 [C. A. 1968, 69, 72393c].

32. Keen, I. M.; Malerbi, B. W. J. Inorg. Nucl. Chem. 1965, 27, 1311.

33. (a) Vacus, J.; Memetzidis, G.; Doppelt, P.; Simmon, J. J. Chem. Soc. Chem. Commun. 1994, 697;

(b) Keen, I. M.; Malerbi, B.W. J. Inorg. Nucl. Chem. 1965, 27, 1311; (c) Barrett, P.A.; Frye, D. A.; Linstead, R. P. J. Chem. Soc. 1938, 1157.

34. (a) Bansho, Y.; Suzuki, S.; Sekiguchi, T.; Saito, I. Kogyo Kagaku Zasshi 1962, 65, 2005; (b) Metz, J.; Schneider, O.; Hanack, M. Inorg. Chem. 1984, 23, 1065. 
35. (a) Berezin, B.D. Izv.Vyssh. Ucheb. Zaved., Khim. Khim. Tekhnol. 1968, 11, 537 [C. A. 1968, 69, 72393c]; (b) Keen , I.M.; Malerbi, B.W. J. Inorg. Nucl. Chem. 1965, 27, 1311; (c) Menzel, E.R.; Rieckhoff, K.E.; Voigt, E.M. Chem. Phys. Lett. 1972, 13, 604.

36. Gurevich, M.G.; Solov'ev, K.N. Dokl. Akad. Nauk. Beloruss. SSR. 1961, 5, 291 [C. A. 1962, 57, 15948].

37. Edward, A.; Cuellar, G.; Marks, J.M. Inorg. Chem. 1981, 20, 3766 .

38. Zhenxiang, L.; Jiazuan, N.; Guangxian, N.; Lemin, L. Wuji. Huaxue. 1986, 2, 8 [C. A. 1987, 106, 202111e].

39. (a) Elvidge, A.; Lever, A.B.P. J. Chem. Soc. 1961, 1257; (b) Meloni, E.G.; Ocone, L.R.; Block, B.P. Inorg. Chem. 1967, 6, 424.

40. Barmasov, A.V.; Korotkov, V.I.; Kholmogorov, V.E. Khim. Fiz. 1986, 5, 414 [C. A. 1992, 116, 197588 r].

Sample Availability: Available from MDPI.

(C) 2001 by MDPI (http://www.mdpi.org). Reproduction is permitted for noncommercial purposes. 